\title{
Effectiveness of Autonomous Learning Materials for Students during the COVID-19 Pandemic: A Case Study of the Daxie Second Elementary School in Ningbo, Zhejiang, China
}

\author{
Zhuodan Xie \\ Daxie Second Elementary School, Ningbo 315812, Zhejiang, China
}

\begin{abstract}
Autonomous learning materials play a vital role in developing students' autonomy. By providing students with appropriate autonomous learning materials to meet their self-diagnosis learning needs, this can effectively improve students' academic performance. During the COVID-19 pandemic, students' learning changed from offline to online. However, in this process, how should teachers and students choose in the face of the rich autonomous learning materials online? This study took students from two elementary schools in Ningbo of Zhejiang Province as the research objects to determine whether autonomous learning materials are conducive to students' autonomous learning. Further, under the same background, answered whether different autonomous learning materials have different effects on student performance. We selected a total of 449 students in the second and fifth grades of the two elementary schools, and after excluding extreme values, 229 were enrolled in the experimental group and 220 were in the control group. The study found that (i) under the influence of COVID-19, the use of autonomous learning materials was effective for most disciplines; (ii) compared with audio and video learning materials, protocolguided learning materials improved students' academic performance much higher. Based on this, we suggest that in the teaching process, teachers should select and use autonomous learning materials in a targeted manner to achieve the purpose of promoting learning.
\end{abstract}


Xie. Autonomous Learning during the COVID-19 Pandemic.

Sci Insigt Edu Front 2020; 6(1):613-624.

Doi: 10.15354/sief.20.or023

How to Cite: Xie, Z. (2020) Effectiveness of autonomous learning materials for students during the COVID-19 pandemic: A case study of the Daxie Second Elementary School in Ningbo, Zhejiang, China. Sci Insigt Edu Front 2020; 6(1):613-624.

Keywords: COVID-19; Autonomous Learning Materials; Elementary School;

Protocol-Guided Learning; Effectiveness

Correspondence to: Zhuodan Xie, Daxie Second Primary School, Ningbo 315812, Zhejiang Province, China. Email: 55479177@qq.com.

Conflict of Interests: None. 
A

UTONOMOUS learning materials are considered to be "learning materials for students with clear teaching goals and learning requirements, free choice of methods, timely feedback, a considerable number of different language levels and language skill practice" (Sheerin, 1991), that is, "with its own design, the learner can directly understand and absorb the contents contained in the textbook to achieve the purpose of learning." This extremely flexible learning material can get rid of the limitations of time and space, cultivate students' independent learning skills and attitudes, adapt to the different experiences, interests, abilities, and needs of individual learners, and then adjust the learning theme, speed, path, depth and breadth accordingly (Yang, 2000).

It is generally believed that the mere existence of autonomous learning facilities does not promote autonomous learning. Autonomous learning materials play a vital role in successfully implementing autonomous learning goals and cultivating students' ability and motivation for autonomous learning. By providing learners with relevant Autonomous learning materials to meet their self-diagnosis learning needs, they have developed into autonomous learners (Cooker, 2008; Morison, 2005).

Since 2010, the Daxie Second Elementary School in Ningbo of Zhejiang Province had independently developed school-based materials suitable for student learning task lists and protocol-guided learning (including the use of task lists for grades one to three, and the use of a protocol for grades four to six). After years of accumulation, the school had formed a relatively complete protocol-guided learning (task list) research, writing, and guidance procedure. Task lists and protocol-guided learning in various disciplines and grades have been taking on a paradigm. During the pandemic period, the school gave a full play to the autonomy and school-based nature of protocol-guided learning (task list), organized teachers to decompose the key and difficult knowledge based on the textbooks, syllabus and various reading materials, and asked different questions and supported with a certain number of targeted practical questions. The formed learning protocol was issued to students so that they can form the habit of selfdirected learning, strive to improve the quality of students' home study, cultivate their self-directed learning ability, and minimize the negative impact of the pandemic on the teaching in the upcoming new semester.

\section{Background}

In 2004, the Association for Educational Communications and Technology (AECT) defined autonomous learning resources as containing people, tools, various technologies, and materials to help students learn. The learning materials were divided into educationpurposed such as traditional textbooks, multimedia courseware, etc. and non-educationpurposed such as electronic encyclopedias, electronic newspapers, software platforms, audiovisual materials, etc. (AECT, 2004).

As the material basis of autonomous learning for students, the choice of learning materials determines students' interest in learning itself, their understanding of knowledge, and the vividness of their learning process (Nan, 2015). Hassan et al. (2012) 
believe that the preparation of Autonomous learning materials designed according to the teaching principles and following the learning content and teaching rules can maximize the learner's academic performance. At the same time, it can enable students to choose their learning strategies according to their characteristics and different learning purposes, cultivate students' self-reflection and self-evaluation awareness and strengthen students' autonomous learning ability (Wang, 2009).

However, "Do different types of learning materials produce different effects for students, and what kind of learning materials are more suitable for students' autonomous learning?" has been becoming a hot topic.

Shi et al. (2016) used 207 seven-grade students from a middle school as objects and used a questionnaire survey to explore the impact of e-schoolbag on students' 1 classroom earning effectiveness. Through the analysis of the pre- and post-test data of the mathematics, it was found that students who used e-schoolbag had significantly higher performance than those who did not use e-schoolbag, indicating that the use of digital learning resources such as e-schoolbag can effectively improve their learning effectiveness.

Besides, the use of video materials such as IMOOC to guide students in an autonomous learning discovery network platform provides a great space for students to communicate independently (Wang, 2017). Deeply aggregated video resources have a significant effect on improving academic performance, curriculum interest, and increasing classroom interactivity (Feng et al., 2016).

In the face of emerging digital learning resources, there are also autonomous learning materials formed by combining traditional paper materials with digital materials, thereby realizing huge innovations in educational materials, promoting the formation of a student-centered teaching model, and ultimately improving the learning flexibility and personalization (Sabrina, 2010). In particular, materials designed and developed by teachers or researchers are more conducive to student learning than those without systematic design (Song \& Sun, 2013).

Most of the above studies have found that the correct use of autonomous learning materials is positively correlated with the improvement of student performance. However, the research results were all based on the normal teaching in the school, as well as the face-to-face guidance of teachers or the counseling agencies. Therefore, it is difficult to say whether the use of autonomous learning materials can also improve students' academic performance without the guidance of teachers and counseling agencies, so the robustness of their data needs to be further improved.

This study carried out during the pandemic isolation. This special event got teachers cannot teach normally, and various counseling agencies also canceled their offline courses, so the above-mentioned two confounding factors were well controlled. The two schools that participated in this study actively responded to the requirements of the pandemic prevention and control, adopted autonomous learning at home. The difference was the choice of autonomous learning materials. The school in the experimental group adopted school-based learning materials, i.e., protocol-guided learning 
materials, while the students in the control group watched the video of famous teachers or live broadcasts and recordings, etc. through the national and local resource platforms.

Therefore, in this study, the Daxie Second Elementary School in Ningbo of Zhejiang adopted protocol-guided learning as its autonomous learning materials as the main intervention variable. Through research, this study aimed to answer the following two questions:

1. Does the use of autonomous learning materials help students improve their academic level without the guidance of teachers and counseling agencies?

2. Do school-based protocol-guided learning and teacher's live recording videos have different effects on students' academic performance?

\section{Research Design}

\section{Objective}

As mentioned above, the use of autonomous learning materials can effectively improve students' academic performance. However, in previous studies, the influence of the two confounding factors like teacher guidance and educational agency counseling could not be ruled out. Therefore, the robustness of their research results needs to be further improved. Based on this, our research, on the one hand, is to verify the effectiveness of students' independent use of the autonomous learning materials during the COVID-19 period; on the other hand, to test whether different autonomous learning materials possess a different effect on their learning outcomes.

\section{Subjects}

We selected the second and fifth-grade students of two elementary schools in Ningbo, Zhejiang Province, China as the subjects. Among them, the Daxie Second Elementary School in Ningbo was used as the experimental group, and the protocol-guided learning materials were prepared by teachers according to the students' learning contents and academic conditions. The control group was another elementary school in Ningbo, and the live broadcasting and recorded video as the autonomous learning materials.

To better control the influence of the students' pre-conditions and pretest differences on the study results, we first investigated the students' family background, parents' educational level and other factors to exclude the interference of these related factors. After obtaining the pre-test results, we matched the experimental data with the control ones and matched the reference sample according to the students' total scores in Chinese and mathematics. After deleting the samples with missing test results, we finally got 229 and 220 valid samples in the experimental and control groups, respectively. The two schools that participated in this study were public schools with a good reputation. No significant differences exist between the two schools in terms of teachers, students, and school conditions. 


\section{Intervention}

In this study, after controlling other related variables, autonomous learning materials were set as the only variable. The experimental school students adopted protocol-guided learning. This is learning material designed by teachers, with students as the main body, problems as the core, practice as the guarantee, and learning method acquisition as the fundamental (Xia, 2020).

To ensure the quality of students' online learning, the control school used teaching videos and live broadcasting videos provided on cloud platforms such as national public resources, local education, and school network as students' autonomous learning materials.

In terms of teaching methods, online teaching has become the only teaching method because classroom teaching cannot be carried out. During the COVID-19 pandemic period, the experimental school organized the teachers of each course to design protocol-guided learning (task list) according to the learning objectives of the textbooks and students' academic conditions to make the knowledge organized and systematic. Teachers will upload protocol-guided learning (task list) to the class group before class, and ask students to prepare according to the protocol and complete the questions. The teacher designs or adjusts the teaching micro-classes according to the situation of the preparation work submitted by the students. After the students have prepared through the protocol, they perform autonomous learning according to the high-quality microclasses uploaded by the teachers and complete the classroom training in the process. After finishing the course, the teacher will arrange a small amount of homework according to the learning situation. Students complete post-practice exercises and upload their homework to class groups, and then checked and appropriately guided by teachers eventually (Xie \& Yang, 2020).

The control school also adopted online teaching and guidance, and arranged students to watch live or recorded video according to the teaching plan or progress every day, and completed the exercises arranged by the teacher in the process, and arranged the exercises according to the learning course after the study. Students uploaded the completed exercises to the online class QQ or WeChat group, and the teachers guided them after the correction.

\section{Test Tools}

The test tools for the pre- and post-tests were all unified standardized test papers prepared by the Teaching and Research Office of Ningbo, Zhejiang Province. The personnel preparing the test papers have rich teaching experience. The test papers compiled by them have shown good reliability and validity in the past years. The pre-test results were selected from the 2019 Ningbo Elementary School unified final exam results, and the post-test results were the city-wide unified test results conducted on April 28, 2020.

In both the experiment and the control groups, student performance was used as an indicator of teaching effectiveness. The students in the experimental group mainly 
used the protocol-guided learning compiled by teachers to conduct targeted autonomous learning. The teacher published the prepared protocol through the online platform, and the students received it and completed it. The teacher timely understood and summarized the completion, and took the problems with a high error rate as the key issues in online classroom teaching. This completed the task of learning while cultivating students' autonomous learning ability. The autonomous learning materials of the students in the control group mainly came from the video of famous teachers from the national resource platform and local education platform or the live broadcasting and recordings of teachers of the school. Through this study, we tried to clarify whether the differences in autonomous learning materials have an impact on students' academic performance.

\section{Research Process}

This study started planning in late January 2020, officially started in early February 2020, and ended in late April 2020.

The end of January 2020 is the preparation stage. At this stage, the two schools first determined the participating students and teachers. Through matching and comparison, the situation of the participating teachers and students was the same, and there was no significant difference. Second, teaching content and progress were coordinated. Finally, the materials needed for the study were prepared and the specific flow of the study was determined.

From February to the end of April 2020, the implementation phase lasted 10 weeks. At this stage, the two schools used different autonomous learning materials for teaching.

\section{Results}

Before the outbreak of the pandemic, all schools in China had finished the 2019 winter semester. Therefore, before the study, the students of both schools used classroom teaching for daily learning. We used the final test scores of the Chinese and Mathematics as the pre-test data. During the pandemic period, students from both schools conducted autonomous learning using different learning materials at home. To verify the effectiveness of autonomous learning materials, the two schools used a unified standardized test paper to test the two subjects of Chinese and mathematics of the two schools as post-test data. All data were analyzed using the SPSS20.0 for independent sample $\mathrm{t}$ test, and the score analysis is shown in Table $\mathbf{1 .}$

From Table 1, the pre-test data of the two groups of second and fifth graders had similar average values in the two subjects of Chinese and mathematics. Among them, the mean value of the fifth-grade Chinese subjects in the control group was higher than that of the experimental group, with a $\mathrm{t}$ value of -0.816 ; and the second-grade mathematics subject had the largest $t$ value of 0.891 , and the average difference between the two groups was about 1.2. The p-values of Chinese and mathematics in the second and fifth grades were both greater than 0.05 . This showed that there were no significant differences between the two groups in the normal teaching environment. 


\begin{tabular}{|c|c|c|c|c|c|c|c|c|c|c|}
\hline & & \multicolumn{4}{|c|}{ Pre-Test } & \multicolumn{4}{|c|}{ Post-Test } & \multirow{2}{*}{$\begin{array}{l}\text { Exp. Effect } \\
\text { Cohen's } d\end{array}$} \\
\hline & & Mean & SD & $t$ & $p$ & Mean & SD & $t$ & $p$ & \\
\hline \multicolumn{11}{|c|}{ 2nd Grade $(\mathrm{Ne}=100, \mathrm{Nc}=95)$} \\
\hline \multirow{2}{*}{ Chinese } & Exp. & 88.31 & 7.96 & \multirow{2}{*}{0.28} & \multirow{2}{*}{0.77} & 77.06 & 16.03 & \multirow{2}{*}{1.18} & \multirow{2}{*}{0.23} & \multirow{2}{*}{-0.21} \\
\hline & Ctrl. & 87.84 & 14.56 & & & 80.00 & 18.54 & & & \\
\hline \multirow{2}{*}{ Math } & Exp. & 91.51 & 7.72 & \multirow{2}{*}{0.89} & \multirow{2}{*}{0.37} & 84.52 & 11.54 & \multirow{2}{*}{3.17} & \multirow{2}{*}{0.002} & \multirow{2}{*}{0.33} \\
\hline & Ctrl. & 90.35 & 10.29 & & & 77.34 & 19.23 & & & \\
\hline \multirow{2}{*}{ Total } & Exp. & 179.82 & 14.15 & \multirow{2}{*}{0.72} & \multirow{2}{*}{0.47} & 161.58 & 24.75 & \multirow{2}{*}{0.95} & \multirow{2}{*}{0.34} & \multirow{2}{*}{0.03} \\
\hline & Ctrl. & 178.18 & 17.27 & & & 157.34 & 36.17 & & & \\
\hline \multicolumn{11}{|c|}{ 5th Grade $(\mathrm{Ne}=129, \mathrm{Nc}=125)$} \\
\hline \multirow{2}{*}{ Chinese } & Exp. & 78.24 & 7.20 & \multirow{2}{*}{0.82} & \multirow{2}{*}{0.42} & 80.27 & 14.69 & \multirow{2}{*}{5.13} & \multirow{2}{*}{0.00} & \multirow{2}{*}{0.75} \\
\hline & Ctrl. & 79.65 & 18.18 & & & 68.03 & 22.61 & & & \\
\hline \multirow{2}{*}{ Math } & Exp. & 80.25 & 17.50 & \multirow{2}{*}{0.39} & \multirow{2}{*}{0.69} & 70.91 & 24.02 & \multirow{2}{*}{9.56} & \multirow{2}{*}{0.00} & \multirow{2}{*}{1.15} \\
\hline & Ctrl. & 79.28 & 21.58 & & & 42.35 & 23.55 & & & \\
\hline \multirow{2}{*}{ Total } & Exp. & 158.49 & 22.56 & 012 & & 140.19 & 40.78 & 570 & 00 & 7 74 \\
\hline & Ctrl. & 158.93 & 32.77 & & & 110.38 & 41.39 & & & \\
\hline
\end{tabular}

Note: Exp.: Experiment Group; Ctrl.: Control Group; SD: Standard Deviation. Ne: Number of the experiment group; Nc: Number of the control group.

Except for the fifth-grade mathematics of the control group (mean: 42.352), all subjects had completed basic learning tasks, and the post-test averages had reached the qualified level (the standard of qualified level means that the average value is greater than or equal to $60 \%$ of the total score). Therefore, it can be concluded that the autonomous learning of students is only effective for some disciplines.

It can also be seen from the post-test results that the two groups had differences in the learning situation of the students in Chinese and mathematics. Compared with the previous average test scores, the second and fifth-grade students had a large difference in the average scores of Chinese and mathematics in the post-test. The fifth-grade students were particularly obvious. Their mathematics t value is 9.562 , and the average difference between the two groups was close to 29. The results of the students in both groups were significantly different $(\mathrm{p}<0.01)$. In the second-grade mathematics, the $t$ value was 3.179 and the $p$-value was 0.002 . There was a significant difference in mathematics between the two groups in between the second graders, with a p-value greater than 0.05 in Chinese. However, overall, the students in the experimental group were generally better than those in the control group who did not use autonomous learning materials.

\section{Discussion}

\section{Under major emergencies, is the use of autonomous learning materials con- ducive to student learning?}


Autonomous learning materials are an important part of autonomous learning equipment. Sufficient autonomous learning materials have a significant positive correlation with learners' language level, needs, and learning style. Well-organized and appropriate autonomous learning materials can promote learners' self-directed learning (Sheerin, 1991).

Compared with the previous test scores, the average post-test scores of the two groups had decreased significantly. The reason for this phenomenon was the lack of autonomous learning ability of elementary school students, especially those from the rural area because they cannot complete the learning tasks independently without the direct guidance of the teacher. According to the data of Balkundi (2006) and Yao (2020), the human capital owned by teachers plays an essential role in changing students' academic performance.

However, we also found that except for the fifth-grade mathematics subject in the control group, and all other post-test scores reached the pass line, indicating that the use of learning materials has a significant impact on student learning without the guidance of teachers. This also demonstrates the idea advocated by Nuthall (2005) that a quarter of the specific concepts and principles of student learning are mainly acquired through the use of learning materials, designing activities by oneself, or collaborating with peers.

Based on this, we believe that under major emergencies, when teacher classroom teaching and guidance cannot be carried out, whether it is a school-based learning resource designed by the teacher or an open network video resource, the proper and reasonable use of autonomous learning materials is conducive to students' academic improvement.

\section{Do different autonomous learning materials have different effects on students' academic performance?}

The two schools participating in this study selected the video resources provided by the national, local, and school cloud platforms and the school-based protocol-guided learning resources prepared by the teachers according to the syllabus and textbooks through collective discussions as the autonomous learning of students during the pandemic period. These were the only variables set in this study.

Previous studies believed that protocol-guided learning fully considered the student's subjective status, and students carried out activities such as learning, questioning, and inquiry-based on the content of the protocol (Chen, 2013), which was conducive to students' timely discovery and feedback of problems, and thus to obtain more targeted guidance, and finally solved the problems in full interaction and communication between teachers and students (Xu \& Du, 2015). Through this study, we found that the experimental group using protocol-guided learning as autonomous learning materials had significantly higher post-test scores than the control one. This result is consistent with the data of Zhou et al. (2020), and further demonstrates the validity of pro- 
tocol-guided learning in previous studies with specific research data as scientific evidence (Ou, 2017).

Based on this, we believe that, compared with other autonomous learning materials, adopting Chinese localized protocol-guided learning based on student subjectivity and learning as student autonomous learning materials is more conducive to the improvement of students' academic level.

\section{Conclusions}

Based on the above results, this study draws the following main conclusions:

\section{Under the influence of COVID-19, when students learn autonomously, the use of autonomous learning materials is effective for most disciplines.}

Through the method of comparative research, this study separately analyzed the students' academic performance in normal teaching and COVID-19 pandemic environments. The results showed that although the overall scores during the epidemic are lower than those in the normal teaching environment, the scores of most of the subjects have reached the qualified level. These data reflect that the use of autonomous learning materials has a certain degree of positive guidance. By relying on their efforts, students can actively improve their ability to learn and explore knowledge.

\section{Compared with audio, video, and other materials, using protocol-guided} learning as autonomous learning materials, students' academic performance has been improved to a higher extent.

A large number of studies reported that protocol-guided learning not only has an important impact on students' academic performance, but also plays a pivotal role in learning ability, independent exploration, and innovation. Zhang et al. (2013) believed that protocol-guided learning, as a student's autonomous learning materials, fully stimulates students' ability to think independently and cooperate, which is a scientific and effective program to promote students' autonomous learning ability.

This study is a further exploration of previous studies. By analyzing the posttest results of the two groups during the pandemic period, it was found that the overall learning performance of the experimental group using protocol-guided autonomous learning materials was significantly higher than the control group using live broadcast and recorded video as autonomous learning materials, which reflects the use of protocol-guided Learning materials can more effectively promote the improvement of students' academic level. Therefore, compared with using live broadcast and recorded video as autonomous learning materials, adopting China's localized protocol-guided learning based on student subjectivity and learning situation as autonomous learning materials is more conducive to the improvement of students' academic level.

\section{Suggestion}


According to the above conclusions, to reasonably choose autonomous learning materials and cultivate students' autonomous learning abilities and motivations, we recommend:

\section{Teachers should be good at using autonomous learning materials.}

The use of autonomous learning materials is not only a wealth of learning materials for students, but also a tool to cultivate students' autonomy and inquiry awareness. Therefore, teachers should make reasonable use of autonomous learning materials so that they can play the largest role in the students' learning process.

\section{Schools and teachers should be targeted in choosing autonomous learning materials.}

The academic conditions of different schools in different regions are different, and the differences in students' cognitive levels are also obvious. Therefore, designing corresponding autonomous learning materials in a targeted manner, with the help of these materials in conjunction with the corresponding autonomous learning mode, can help students improve their academic performance.

\section{References}

AECT. (2004) Definition and technology committee document. http: Indiana edu/molpage/Meaning s\% 20of\%20ET _4.0.pdf. June1, 2004.

Balkundi, P., \& Harrison, D. (2006) Ties, leaders, and time in teams: Strong inference about network structure's effects on team viability and performance. Acad Manag J, 49(5):49-68.

Chen, L. (2013) Exploring and Thinking of the "Guidance Study Case" Teaching Model in Secondary Vocational English Class. Qizhi Weekly, (08):27-28.

Cooker, L. (2008). A history of the SALC in the ELI handbook 2008-2009. Makuhari: KUIS.

Feng, S., Wang, X., \& Zhang, S. (2016) Research on design and application of video resource aggregation model based on knowledge element. Chin Audiovis Edu, 37(9):88-94.

Hassan, H., Hassan, F., Omar, N.D., Zakaria, Z., \& Nor, W.A.W.M. (2012). Evaluating mathematics e-learning materials: do evaluators agree with distance learners? Procedia Soc Behav Sci, 67:189-195.

Morison, B.R. (2005) A framework for the evaluation of a self-access language learning centre. Supporting independent English language learning in the 21 st century: proceedings of the independent learning association conference inaugural, Manukau Institute of Technology, Auckland.

Nan, X. (2015) The selection of "materials" is more exciting-thinking about the inefficiency of learning materials and selection strategies. Math Learn Res, (10):68. 
Nuthall, G.A. (2005) The cultural myths and realities of classroom teaching and learning: A personal journey. Teacher Coll Rec, 107(5):895-934.

Ou, F. (2017) An empirical study of the tutoring case in high school English reading teaching. Guiyang: Guizhou Normal University.

Sabrina, L., \& Tommaso, L. (2010) The synergy of paper-based and digital material for ubiquitous foreign language learners. Knowl Manag E-Learn, 3.

Sheerin, S. (1991) Self-access. Lang Teach, (3):143-157.

Shi, Y., Yang, H., Wu, Y., Zhang, X., \& Wei, S. (2016) Analysis of the actual effect of information-based learning terminals in classroom teaching: Taking middle school mathematics as an example. Chin Audiovis Edu, (07):87-92.

Song, W., \& Sun, Z. (2013) Meta-analysis of the effectiveness of digital learning resources. Chin Teleph Edu, (11):81-85.

Wang, W. (2017) Study on the effectiveness of using IMOOC resources to guide students' self-directed learning to improve students' learning ability. J Jiamusi Vocat Coll, (09):309.

Wang, Y. (2009) Action Research on cultivating adult students' independent learning ability based on independent learning materials. Ningbo: Ningbo University.
Xia, J.P. (2020) Teaching for student learning: Exploration of teaching strategies based on protocol-guided learning. Sci Insigt Edu Front, 5(1):451-457.

Xie, Z.D., \& Yang, J.N. (2020) Autonomous learning of elementary students at home during the COVID-19 epidemic: A case study of the Second Elementary School in Daxie, Ningbo, Zhejiang Province, China. Best Evid Chin Edu, 4(2):535-541.

Xu, F., \& Du, Q. (2015) Optimization of "problem design" in the political guidance case of high school. Teach Manag, (19):66-67.

Yang, J. (2000) Self-study textbook design manual. (Taiwan) Psychological Publishing House, pp3-pp5.

Yao, J.J., Rao, J.L., Jiang, T., \& Xiong ,C.Q. (2020) What role should teachers play in online teaching during the COVID-19 pandemic? Evidence from China. Sci Insigt Edu Front, 5(2):517-524.

Zhang, Y., \& Meng, Z. (2013) The value, limits and symbiosis of "Guidance Case Teaching" and "Flip Class". Glob Edu Outlook, 42(07): 10-17+94.

Zhou, L.J., \& Li, C.G. (2020) Can student self-directed learning improve their academic performance? Experimental evidence from the instruction of protocolguided learning in china's elementary and middle schools. Sci Insigt Edu Front, 5(1):469-480. 